\title{
Sodium valproate and routine liver function tests
}

There has been concern in recent years about the adverse effects of sodium valproate and in particular over deaths reported to be due to hepatotoxicity. The histological findings are most commonly those of microvesicular steatosis. ${ }^{1}$ As most deaths have been in children or young adults ${ }^{2}$ and sodium valproate is frequently prescribed in childhood epilepsy, it is important that paediatricians should be aware of the pathogenetic mechanisms underlying the hepatotoxicity and whether or not such events are preventable or at least predictable. Rolles, ${ }^{3}$ in his review of 49 fatal cases of hepatotoxicity caused by sodium valproate, analysed in detail the childhood fatalities reported in the United Kingdom. All of the seven children in this series had some pre-existing problem in addition to epilepsy; three or four had degenerative disease, four or five had developmental delay, and two had hepatomegaly. Most had refractory myoclonic epilepsy. All were on combinations of anticonvulsant drugs at the time of presentation and all the severe reactions occurred within 10 weeks of starting treatment. Rolles postulated four possible mechanisms for the hepatotoxic reaction:

(a) No relation at all-possible but unlikely in view of the evidence.

(b) A direct toxic relation-this is unlikely in view of the very large number of cases (over a million) that have been treated with relatively few fatalities and the absence of any dose related effect.

(c) Intercurrent infection, for example, hepatitis modified by sodium valproate-again there is little evidence to support this as there have been recorded outbreaks of hepatitis in institutions where children are on sodium valproate, without strong evidence of either clinical or biochemical hepatotoxicity. ${ }^{4}$

(d) An idiosyncratic reaction to sodium valproate in a small group of children who have either specific metabolic problems or other predispositions. Rolles felt that this was the most likely answer and this is supported by subsequent reports. ${ }^{25}$

Assuming an idiosyncratic reaction, it would be helpful if there were some form of biochemical marker which indicated that a particular child was predisposed to such a reaction. Erasmus, Hjelm, and Wilson ${ }^{6}$ studied this idea in a series of children taking sodium valproate and other anticonvulsants. Although concentrations of aspartate amino trans- ferase and alanine amino transferase were raised in children taking sodium valproate, the values were not significantly different from those in children taking other anticonvulsants and on repeat estimations had fallen to normal. Raised values could be explained by intercurrent infection or by the effect of enzyme induction when anticonvulsant drugs are started. No evidence of any consistent trend to hepatotoxicity in children taking sodium valproate was found. Similar results are reported by Rochel and Ehrenthal ${ }^{7}$ who also measured alkaline phosphatase and $\gamma$ glutamyltranspeptidase and found these were raised only in patients taking other drugs in addition to sodium valproate. As ammonia can also be raised in the absence of hepatotoxicity ${ }^{8}$ in children taking sodium valproate, liver function tests may in fact be misleading and of little predictive value.

Both Rolles ${ }^{3}$ and Jeavons ${ }^{2}$ concluded that it would seem prudent to take particular care when using sodium valproate in those children with known neurometabolic disease or progressive epilepsy, particularly of a myoclonic nature, or to avoid it where there was a family history of a reaction to valproate.

All anticonvulsants have adverse effects and some may give rise to hepatotoxicity. Sodium valproate is an effective anticonvulsant if used appropriately; the risk of hepatotoxicity is small and if the child has been on treatment for more than six months a hepatotoxic reaction is very unlikely indeed. In children taking the drug clinical awareness and vigilance are the most effective way of detecting any hepatotoxicity. If unexplained signs of lethargy, vomiting, or general deterioration occur the drug should be stopped.

A case has been reported ${ }^{9}$ of a child taking sodium valproate and clonazepam who died of fatal hepatotoxicity; he was found to be heterozygous for $\alpha_{1}$ antitrypsin and it was postulated that this protease deficiency made the child more vulnerable to sodium valproate. It remains to be seen if this or other specific markers will be of help in the future-but at the moment routine liver function tests do not seem to have any value in predicting an idiosyncratic reaction to sodium valproate.

\footnotetext{
References

1 Zimmerman HJ, Ishak KG. Valproate-induced hepatic injury: analyses of 23 fatal cases. Hepatology 1982;2:591-7.
} 


\section{Green}

2 Jeavons PM. Non-dose-related side effects of valproate. Epilepsia 1984;25(Suppl 1):S50-5.

${ }^{3}$ Rolles CJ. Hepatic injury with sodium valproate: a reappraisal with special reference to children. Br J Clin Pract [Symp Suppl] 1983;27:72-6.

${ }^{4}$ Dowling St J. Hepatitis A in three patients taking sodium valproate. Lancet 1984;i:963-4.

5 Schnabel R, Rambeck B, Janssen F. Fatal intoxication with sodium valproate. Lancet 1984;i:221-2.

${ }^{6}$ Erasmus C, Hjelm M, Wilson J. The value of routine liver function monitoring during sodium valproate therapy. Br J Clin Pract [Symp Suppl] 1983;27:77-8.
${ }^{7}$ Rochel M, Ehrenthal W. Effect of valproic acid on hepatic $\bigcirc$ function. In: Oxley J, Janz D, Meinardi H, eds. Chronic toxicity of antiepileptic drugs. New York: Raven Press, 1983:61-8.

8 Anonymous. Drugs for epilepsy. Med Lett 1983;25:81-4.

${ }^{9}$ Moore JR, Williams THC, Talbot IC, Tanner MS. Heterozygous $\alpha 1$ antitrypsin deficiency and valproate hepatotoxicity. Lancet 1984;i:221.
S H GREEN University of Birmingham, Institute of Child Health, Birmingham 